\title{
LIG System for Word Level QE Task at WMT14
}

\section{INTRODUCTION}

口 Task 2, WMT14: Word-level Confidence Estimation New point: MT outputs are collected from multiple translation means (machine and human).

$\checkmark$ Our approach: (Figure 1)

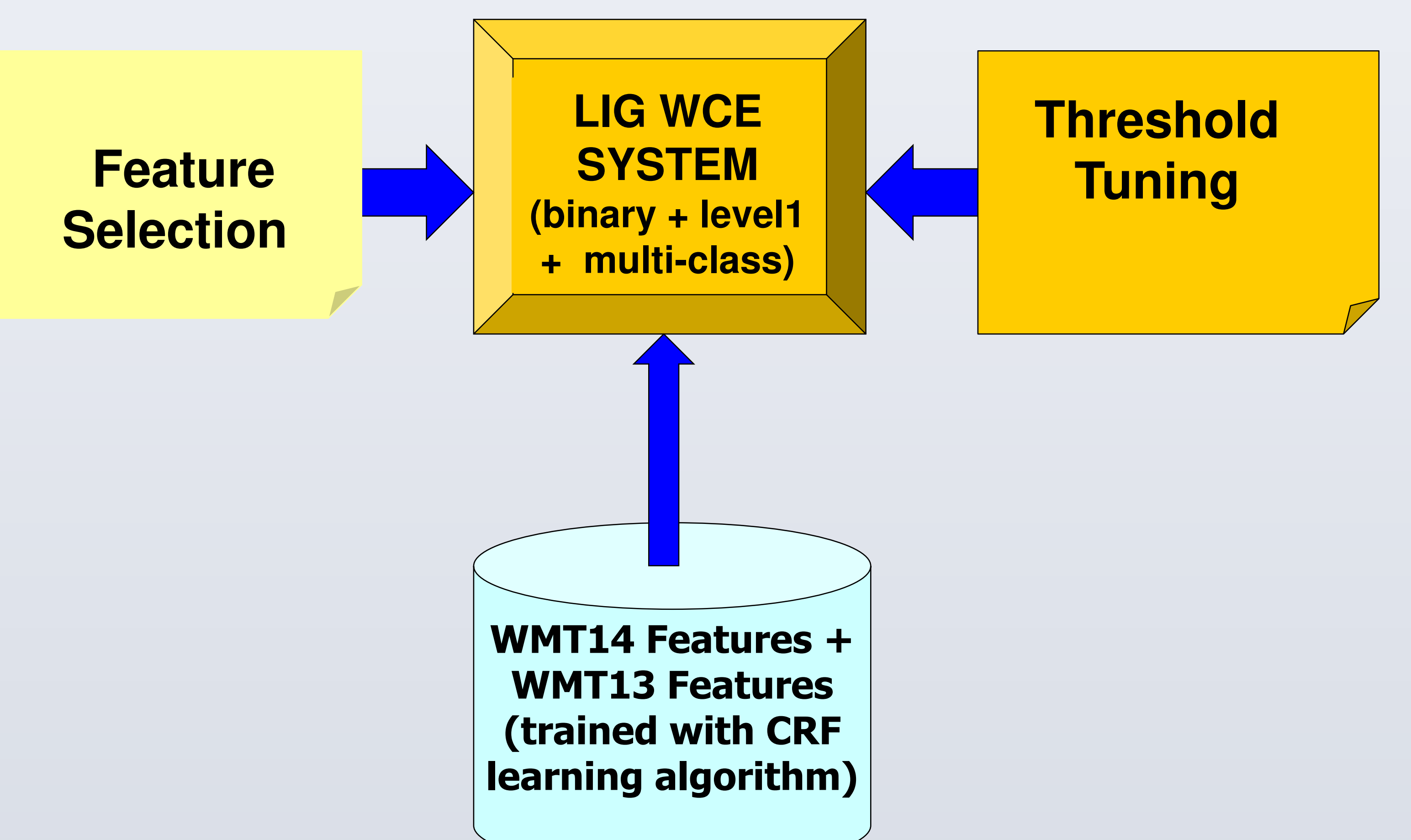

Figure 1: LIG approach for Task 2, WMT 2014

\section{FEATURE TYPES (24 IN TOTAL)}

वThe conventional features (Table 3): work efficiently in our WMT13 submissions and are inherited in this year's systems.

口The WMT14 features (bold and italic in Table 3): are more specifically suggested by us for this year.

\section{EXPERIMENTAL SETTINGS}

\section{AND PRELIMINARY EXPERIMENTS}

DData sets: Description (Figure 2), Statistics (Table 1)

$$
\begin{array}{ccc}
\text { Training } \\
\text { set: 1957 } \\
\text { MT outuputs } \\
\text { + labels }
\end{array}
$$

WMT14 DATA SETS LIG DATA SETS

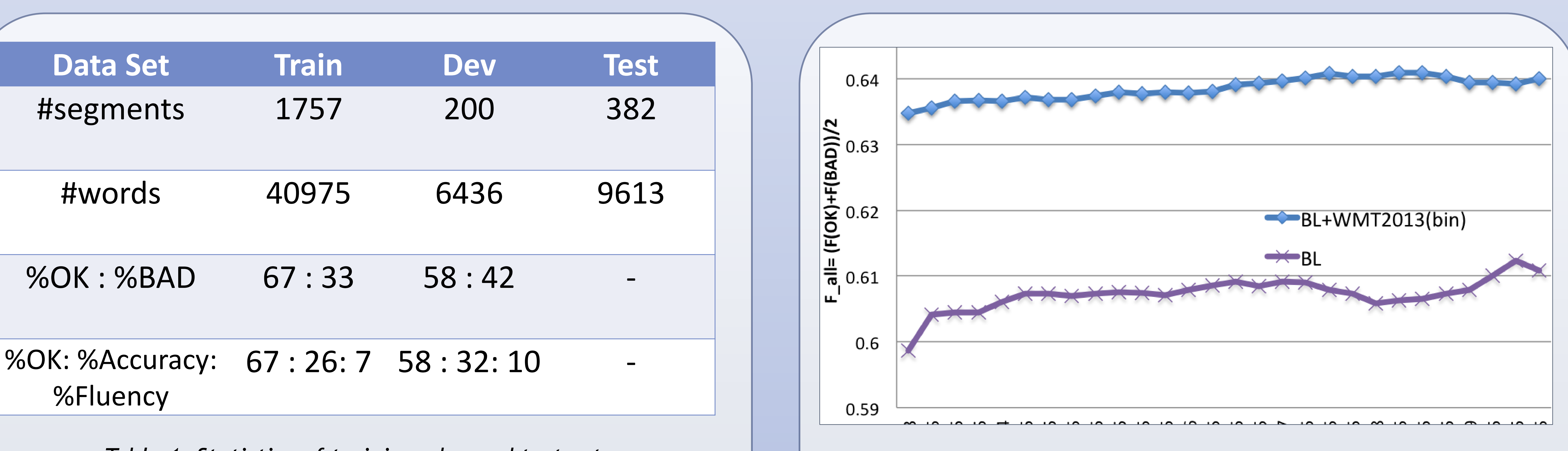

Table 1: Statistics of training, dev and test sets.

WMT13 data is used to combine with WMT14

data in binary variant.

Machine learning method: Conditional Random Fields (CRF).

Toolkit for training and labeling: WAPITI.

Gor binary system: the classification corresponds to a threshold increase from 0.300 to 0.975 (step $=0.025$ ) (Figure 3). Optimal value $=0.75$.

DResults: BL BIN, BL L1, BL MULT and

BL+WMT13 BIN in Table 2 FEATURE SELECTION (FS)

- Objectives: filter the most informative features,

eliminate the useless ones.

- Sequential Backward Selection

- Best systems : FS_BIN, FS_L1, FS_MULT (Table 2).

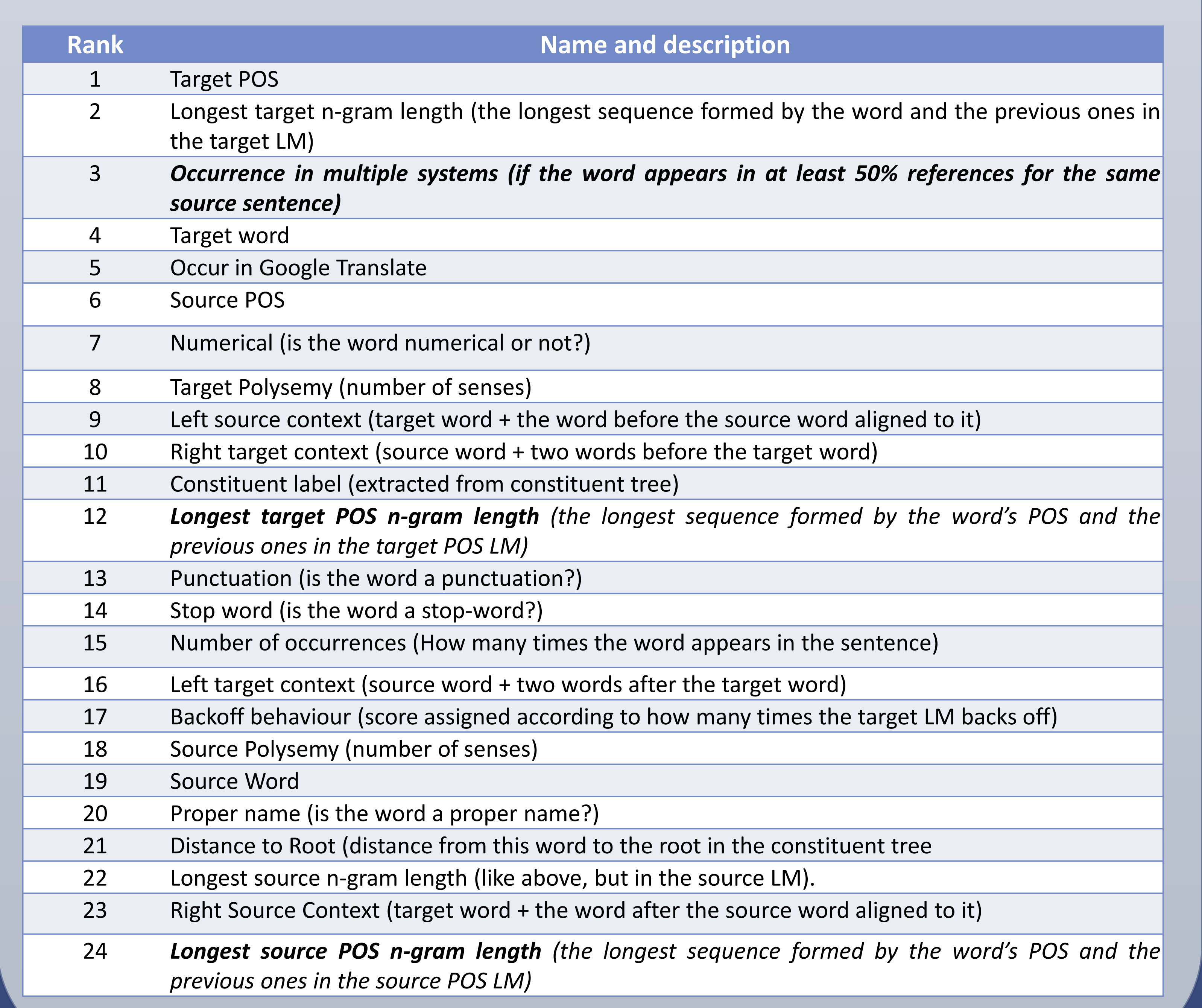

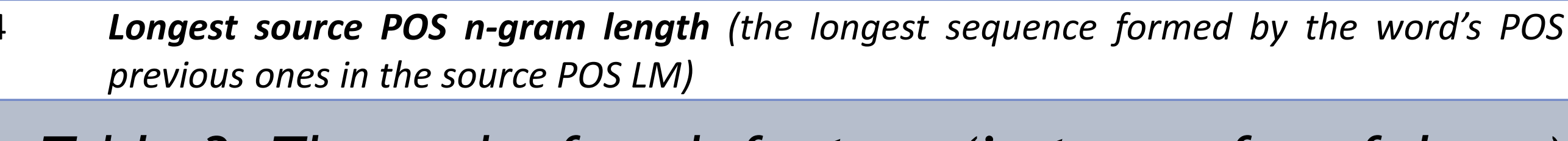
Table 3: The rank of each feature (in term of usefulness).

\begin{tabular}{|c|c|c|c|c|}
\hline System & Label & $\operatorname{Pr}(\%)$ & $\mathrm{Rc}(\%)$ & $F(\%)$ \\
\hline \multirow{2}{*}{ BL_BIN } & OK & 66.67 & 81.92 & 73.51 \\
\hline & BAD & 60.69 & 41.92 & 49.58 \\
\hline \multirow{3}{*}{ BL_L1 } & OK & 63.86 & 82.83 & 72.12 \\
\hline & Accuracy & 22.14 & 14.89 & 17.80 \\
\hline & Fluency & 50.40 & 27.98 & 35.98 \\
\hline BL_MULT & All labels & \multicolumn{3}{|c|}{ Favg(all) $=24.84$} \\
\hline \multirow{2}{*}{$\begin{array}{c}\text { BL+WMT13 } \\
\text { _BIN }\end{array}$} & OK & 68.62 & 82.69 & 75.01 \\
\hline & BAD & 64.38 & 45.73 & 53.47 \\
\hline \multirow{2}{*}{ FS_BIN } & OK & 68.89 & 83.14 & 75.35 \\
\hline & BAD & 64.66 & 46.37 & 54.00 \\
\hline \multirow{3}{*}{ FS_L1 } & OK & 64.03 & 83.47 & 72.47 \\
\hline & Accuracy & 22.44 & 15.68 & 18.46 \\
\hline & Fluency & 51.71 & 27.67 & 36.05 \\
\hline FS_MULT & All labels & \multicolumn{3}{|c|}{$\operatorname{Favg}($ all $)=24.88$} \\
\hline
\end{tabular}

Figure 3: Decision threshold tuning on BL(bin) and BL+WMT2013(bin) Table 2: Pr, Rc and $F$ for labels of all binary, level 1 and multi-class
systems, obtained on dev set.
aPerformance Evolution during FS (Figure 4) Best subset: Top 18

$\square$ Best proposed feature: Occurrence in mult. systems

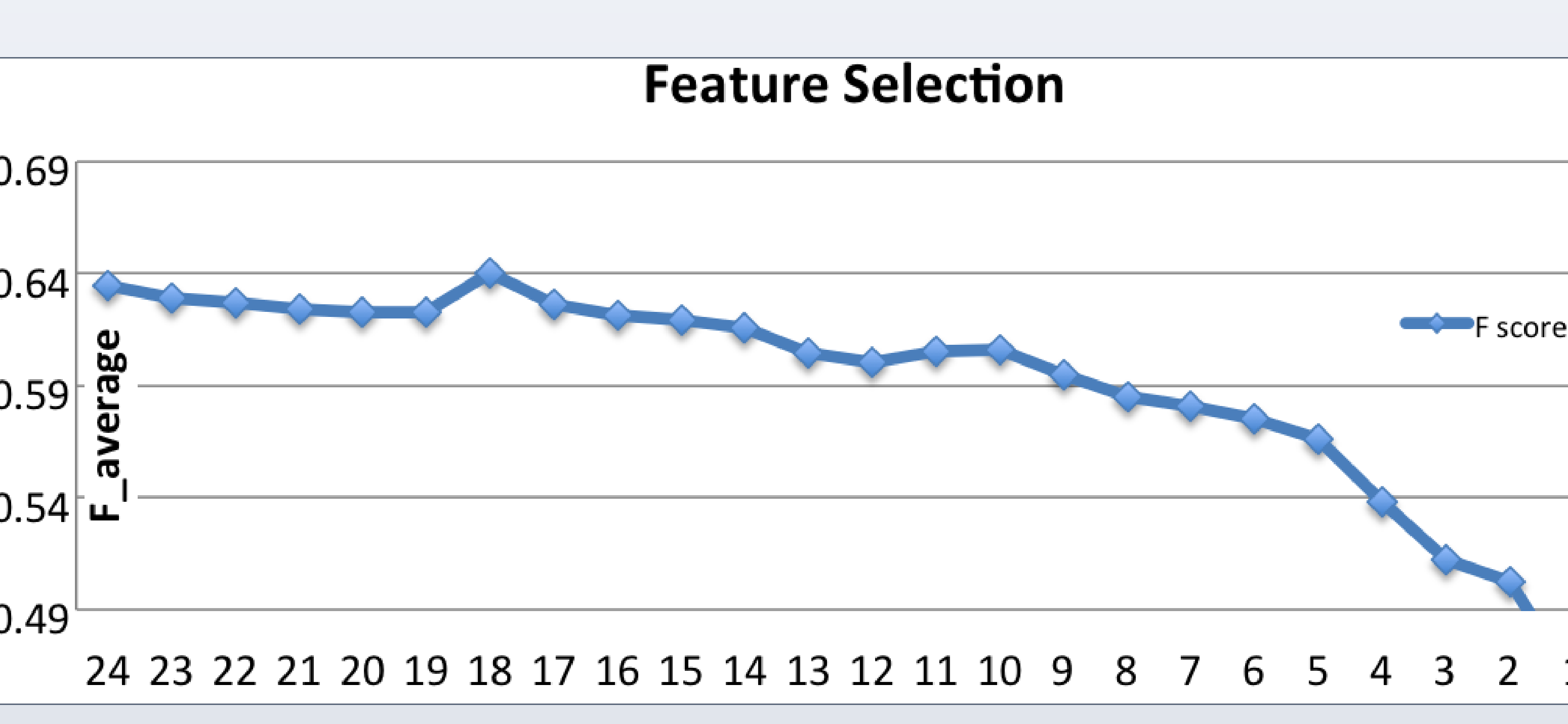
Figure 4: Evolution of system performance (Favg (all)) during

Feature seletion por system partor

\section{SUBMISSIONS AND OFFICIAL RESULTS}

$\square$ Average $F$ (main metric): average $F 1$ for all but the 'OK' class.

\begin{tabular}{|c|c|c|}
\hline System & Average $\mathrm{F}(\%)$ & $\mathrm{F}\left({ }^{\left(O K^{\prime}\right)}\right)(\%)$ \\
\hline $\begin{array}{l}\text { FS_BIN } \\
\text { (primary) }\end{array}$ & 44.4735 & 74.0961 \\
\hline FS_L1 & 31.7814 & 73.9856 \\
\hline FS_MULT & 20.4953 & 76.6645 \\
\hline BL+WMT13(BIN) & 44.1074 & 74.6503 \\
\hline BL_L1 & 31.7894 & 74.0045 \\
\hline BL_MULT & 20.4953 & 76.6645 \\
\hline
\end{tabular}

aF('OK'): F1 for 'OK' class.

Table 4: Official results of the submitted systems. CONCLUSIONS AND PERSPECTIVES DIntegration of several novel features.

aFeature Selection's help to enlighten the valuable features.

MMore data (WMT13) helps to boost performance

afuture work: research linguistic features, reinforce the segment- level CE, propose the methodology for Sentence CE relied on the word- and segment- level. 